\title{
Dangers of long waiting times for outpatient appointments at a urology clinic
}

\author{
K German, F Nuwahid, P Matthews, \\ T Stephenson
}

\begin{abstract}
Department of Urology, Cardiff Royal Infirmary, Cardiff CF2 1SZ

$\mathrm{K}$ German, research fellow in urology

F Nuwahid, honorary clinical attachment in urology P Matthews, consultant urologist

T Stephenson, consultant
\end{abstract} urologist

Correspondence to:

Mr German.

BMF 1993;306:429
Great pressure has recently been put on clinicians by hospital managers and politicians to reduce waiting times. Unfortunately, the emphasis of current initiatives on waiting lists tends to be on reducing the wait for surgery rather than the waiting time for an appointment at an outpatient clinic. We report the potential dangers of long waiting times for a routine outpatient appointment at a urology clinic.

\section{Patients, methods, and results}

Over the past three years 55 patients with symptoms of bladder outflow obstruction were recruited for two clinical trials. These patients were recruited from the waiting list of new patients which comprised patients who had been classified as having routine conditions by the consultant on the basis of the information in the referral letter. Recruitment into the trials depended on patients satisfying the entry criteria and giving their informed consent. The protocols for the trials were approved by the ethical committee. The average wait for these patients who were seen outside the normal times of outpatient clinics, was 13 (range 3-104) weeks. All patients gave a full medical history and had a full examination, and routine investigations for bladder outflow obstruction were performed. One trial (25 patients) also required transrectal ultrasonography of the prostate and a serological test for prostate specific antigen whereas the other (30 patients) required further investigation only if malignancy was suspected clinically.

During our investigation of these patients, we diagnosed seven new cases of cancer of the prostate. These were detected by rectal examination (five), a raised concentration of prostate specific antigen (one), and transrectal ultrasonography (one). Four of these cancers were well or moderately well differentiated, and five of the six bone scans obtained yielded negative results. A superficial cancer of the bladder was detected during transrectal ultrasonography of the prostate and a caecal cancer was found on barium enema examination in a patient with iron deficiency anaemia. In four patients a history of haematuria was elicited for the first time and was investigated. Ultrasonography also detected one case of chronic retention of urine with overflow.

\section{Comment}

This study highlights the prevalence of associated disease in patients who were classed as having routine bladder outflow obstruction. The average wait for a routine appointment at our department's outpatient clinic was eight months, with a further wait of two years for a prostatectomy. A random poll of 16 departments of urology was conducted by means of a telephone conversation with each consultant's secretary or the clerk responsible for admissions. The average waiting time for a routine appointment in a urological outpatient clinic was $7 \cdot 4$ (range $3-36$ ) months and for a non-urgent prostatectomy 21 (9-36) months, and the situation in Wales was slightly worse than in the United Kingdom as a whole.

Opinion is divided about the value of screening for prostatic cancer and about the management of localised disease. Since $84 \%$ of apparently localised cancers of the prostate progress if left untreated ${ }^{1}$ some centres advocate radical prostatectomy for early cancer. ${ }^{23}$ If long term follow up shows improved survival in patients treated by radical surgery then early detection of the tumour by screening would be desirable, and the most useful method of detection is rectal examination by a trained urologist. ${ }^{4}$ Since most of the prostatic cancers detected in this study had not metastasised to bone and were well or moderately well differentiated these patients might have suffered if detection had been delayed.

A long wait for a patient with bladder outflow obstruction for a specialist opinion is both undesirable and unacceptable. The high prevalence of associated disease in these patients should encourage and justify efforts to reduce waiting times for appointments at outpatient clinics.

1 George NJ. Natural history of localised prostatic cancer managed by conservative therapy alone. Lancet 1988;i:494-7.

2 Steinberg GD, Epstein JI, Piantadosi S, Walsh PC. Management of stage D1 adenocarcinoma of the prostate: the Johns Hopkins experience 1974 to 1987. f Urol 1990;144:1425-32.

3 Zincke $H$. Extended experience with surgical treatment of stage D1 adenocarcinoma of the prostate. Significant influences of immediate adjuvant hormonal treatment (orchiectomy) on outcome. Urology 1989;33 (suppl 5):27-36.

4 Perrin P, Marquet JH, Bringeon G, Devonec $M$. Screening for prostate cancer. Comparison of trans-rectal ultrasound, prostate specific antigen and rectal examination. Br f Urol 1991;68:263-5.

(Accepted 21 September 1992)

\section{Effect of long term tamoxifen treatment on bone turnover in women with breast cancer}

\author{
C D P Wright, R E Mansell, J-C Gazet, \\ JE Compston
}

The non-steroidal antioestrogen tamoxifen is widely used to treat breast cancer, predominantly in postmenopausal women. It exhibits oestrogenic and antioestrogenic properties, depending on the species and tissue. In the human breast it acts as an antioestrogen whereas oestrogenic effects have been reported on human vaginal epithelium and endometrium. ${ }^{1}$ If tamoxifen exerts antioestrogenic effects on bone women receiving long term treatment may be at greater risk of osteoporotic fracture. Densitometry has generally shown no adverse effect of tamoxifen on bone mass in postmenopausal women, ${ }^{2}$ although one study reported loss of bone in premenopausal women. ${ }^{3}$ Our study provides the first histological data on bone turnover in women receiving long term tamoxifen treatment.

\section{Patients, methods, and results}

Forty one postmenopausal women aged 40-70 with stage I or II breast cancer were recruited into the study on the basis of their willingness to undergo bone biopsy; 22 had received tamoxifen for at least 15 (range 15-54) months, and the remainder had not received tamoxifen. Women with a history of bone disease and those taking drugs or with conditions known to affect bone metabolism were excluded from the study.

Full thickness biopsy specimens of the iliac crest were obtained under local anaesthesia after the women 
Department of Medicine, University of Cambridge Clinical School,

Addenbrooke's Hospital, Cambridge CB2 2QQ

C D P Wright, research associate

J E Compston, honorary consultant physician

Department of Surgery, University of Wales College of Medicine, Cardiff CF4 4XN

R E Mansell, professor of surgery

Department of Surgery, St George's Hospital, London SW17

$\mathrm{J}-\mathrm{C}$ Gazet, consultant surgeon

Median (interquartile range) histomorphometric variables of bone in postmenopausal women with breast cancer receiving tamoxifen and controls

\begin{tabular}{|c|c|c|c|c|}
\hline & Bone area $(\%)$ & Osteoid perimeter (\%) & Mineralising perimeter $(\%)$ & $\begin{array}{l}\text { Rate of formation of bone } \\
\left(\mu \mathrm{m}^{2} / \mu \mathrm{m} / \text { day }\right)\end{array}$ \\
\hline $\begin{array}{l}\text { Women with breast cancer: } \\
\text { Receiving tamoxifen } \\
\text { Not receiving tamoxifen } \\
\text { Normal women' }\end{array}$ & $\begin{array}{l}23 \cdot 2(19-25 \cdot 5) \\
20 \cdot 9(18 \cdot 5-24 \cdot 3) \\
20 \cdot 8(14-30)\end{array}$ & $\begin{array}{l}16 \cdot 7(9 \cdot 9-24 \cdot 2) \\
14 \cdot 3(10 \cdot 9-17 \cdot 9) \\
12 \cdot 8(7-25)\end{array}$ & $\begin{array}{l}3 \cdot 7(2 \cdot 7-6 \cdot 4) \\
4 \cdot 5(3 \cdot 3-9 \cdot 1) \\
6 \cdot 1(0 \cdot 5-8)\end{array}$ & $\begin{array}{l}0.028(0.02-0.036)^{\star} \\
0.049(0.027-0.066)^{\star} \\
0.038(0.003-0.068)\end{array}$ \\
\hline
\end{tabular}

*Women with breast cancer receiving tamoxifen $v$ not receiving tamoxifen, $p=0 \cdot 05$. All other comparisons not significant.

had received two separate doses of demeclocycline, and a venous blood sample was taken. Bone histomorphometry was performed by image analysis. The rate of formation of bone was calculated as the rate of mineral apposition $\times$ (half the surface single labelled with demeclocycline + all the double labelled surface (expressed as a percentage of the total surface)). ${ }^{4}$ The groups were compared with the Mann-Whitney test.

The median age of the women taking tamoxifen was 56 (range 40-70) and the time since the menopause 6 $(0-25)$ years. The control group's median age was 64 (46-69) and the time since the menopause $15(0-29)$ years. These values were not significantly different between the groups. Median body weight, however, was significantly higher in the women taking tamoxifen $(68.9 \mathrm{~kg})$ than in the controls $(61.4 \mathrm{~kg})(\mathrm{p}<0.02)$. The median serum alkaline phosphatase concentration in the women taking tamoxifen was 60.0 (interquartile range 53-68) $U / 1$ compared with 86.5 (68-110) $U / 1$ in the controls $(p<0.005)$. There were no significant differences in serum concentrations of urea, electrolytes, calcium, phosphate, bilirubin, alanine aminotransferase, or parathyroid hormone. The median rate of tissue based bone formation in the patients treated with tamoxifen was lower than that in the controls (table). There were no significant differences between the groups in bone area, osteoid perimeter, or mineralising perimeter (table), and $95 \%$ of these values lay within the ranges reported for normal postmenopausal women. ${ }^{5}$

\section{Comment}

These results show that long term tamoxifen treatment does not adversely affect bone turnover in women with breast cancer. The preservation of normal trabecular bone area in our patients is consistent with most densitometric data, which indicate either neutral or oestrogenic effects of tamoxifen on bone. ${ }^{2}$ The significantly lower median serum alkaline phosphatase concentration in the women treated with tamoxifen is consistent with an oestrogenic effect of the drug since none of the women had demonstrable hepatic or skeletal metastases at the time of bone biopsy. The lower rate of tissue based bone formation in these patients is also consistent with this hypothesis: if tamoxifen had antioestrogenic effects an increased rate of bone formation would be expected.

Our results thus provide the first direct evidence that tamoxifen does not have antioestrogenic effects on bone in postmenopausal women and indicate a possible oestrogenic effect.

This study was funded by the Cancer Research Campaign. JEC is supported by the Wellcome Trust.

1 Fornander T, Rutqvist LE, Cedermark B, Glas U, Mattsson A, Silfversward C et al. Adjuvant tamoxifen in early breast cancer: occurrence of new primary cancers. Lancet 1989; i:117-20.

2 Love RR, Mazess RB, Barden HS, Epstein S, Newcomb PA, Jordan VC, et al. Effects of tamoxifen on bone mineral density in postmenopausal women with breast cancer. N Engl I Med 1992;326:852-6.

3 Gotfredsen A, Christiansen C, Palshof T. The effect of tamoxifen on bone mineral content in premenopausal women with breast cancer. Cancer mineral conten

4 Parfitt AM, Drezner MK, Glorieux FH, Kanis JA, Malluche HM, Meunier PJ, et al. Bone histomorphometry: standardization of nomenclature, symbols and units. Report of the ASBMR histomorphometry nomenclature committee. f Bone Miner Res 1987;2:595-610.

5 Recker RR, Kimmel DB, Parfitt AM, Davies KM, Keshawarz N, Hinders S Static and tetracycline-based bone histomorphometric data from 34 normal postmenopausal females. $\mathcal{f}$ Bone Miner Res 1988;3:133-45.

(Accepted 30 September 1992)

\section{Prevalence of symptoms in patients with simple renal cysts}

\author{
Alfredo Caglioti, Ciro Esposito, Giorgio Fuiano, \\ Carlo Buzio, Maurizio Postorino, \\ Teresa Rampino, Giuseppe Conte, \\ Antonio Dal Canton
}

A few anecdotal reports have indicated simple renal cysts as a possible cause of hypertension, flank pain, macroscopic haematuria, and erythrocytosis. ${ }^{1-3}$ Many physicians also believe that simple renal cysts commonly cause microscopic haematuria and mild proteinuria. The frequency of association of simple renal cysts with any of these symptoms, however, has never been the object of controlled epidemiological study, and certain important questions therefore lack definite answers. For example, should further diagnostic procedures (such as renal biopsy) be performed, after simple renal cysts are found in a patient with mild proteinuria or microscopic haematuria? Is the prevalence of flank pain or macroscopic haematuria in patients with simple renal cysts so high as to justify invasive procedures (such as removal by surgery or the application of alcohol)?

\section{Methods and results}

Simple renal cysts were defined as one or a few cysts of $0.2 \mathrm{~cm}$ in diameter or more that did not result from adult polycystic disease, medullary cystic disease, or pyelogenic cysts. The diagnosis was based on ultrasonography.

In our cooperative study, in five general hospitals, renal ultrasonography was scheduled in 1526 consecutive patients who had been referred to the ultrasound service for any abdominal examination. Patients aged under 18 , bedridden patients, and patients with renal failure or a history of kidney transplantation were excluded. Two hundred and eighty four patients were classed as renal patients because the disease causing admission-as recorded on their official case sheetwas renal or related to the urinary tract; the remaining 1243 were classed as non-renal patients.

Medical data were recorded shortly before the renal ultrasonographic examination, and included age, sex, admission diagnosis, and a history of episodes of colicky pain or tenderness in the flank, macroscopic haematuria, and hypertension. The renal ultrasonographic findings were recorded by the radiologist and included renal cysts - their site, number, and diameter. Urine analysis, a red blood cell count, and blood pressure were also routinely recorded.

Analysis of variance and an unpaired $t$ test were used 